\title{
Interrelation of preventive care benefits and shared costs under the Affordable Care Act (ACA)
}

\author{
Robert Brent Dixon*, Attila J. Hertelendy
}

Abstract

With the implementation of the Affordable Care Act (ACA), access to insurance and coverage of preventive care services has been expanded. By removing the barrier of shared costs for preventive care, it is expected that an increase in utilization of preventive care services will reduce the cost of chronic diseases. Early detection and treatment is anticipated to be less costly than treatment at full onset of chronic conditions. One concern of early detection of disease is the cost to treat. In reality, the confluence of early detection may result in greater overall expenditures. Even with improved access to preventive care benefits, cost-sharing of other health services remains a major component of insurance plans. In order to treat identified conditions or diseases, cost-sharing comes into play. With the greater adoption of cost-sharing insurance plans, expenditures on the part of enrollee are anticipated to rise. Once the healthcare recipients realize the implication of early identification and resultant treatment costs, enrollment in preventive care may decline. Healthcare legislation and regulation should consider the full spectrum of care and the microeconomic costs associated with preventive treatment. Although the system at large may not realize the immediate impact, behavioral shifts on the part of healthcare consumers may alter healthcare. Rather than the current status quo of treating presenting conditions, preventive treatment is largely anticipated to require more resources and may impact the consumer's financial capacity. This report will explore how these two concepts are co-dependent, and highlight the need for continued reform.

Keywords: Preventive Care, Affordable Care Act (ACA), Shared Costs, Cost Management, Insurance Accessibility Copyright: @ 2014 by Kerman University of Medical Sciences

Citation: Dixon RB, Hertelendy AJ. Interrelation of preventive care benefits and shared costs under the Affordable Care Act (ACA). Int J Health Policy Manag 2014; 3: 145-148. doi: 10.15171/ijhpm.2014.76
Article History:

Received: 24 July 2014 Accepted: 22 August 2014 ePublished: 23 August 2014

*Correspondence to: Robert Brent Dixon Email: dixon@gwu.edu

\section{Statement of the issue and background}

The need for healthcare access and cost management of healthcare programs is essential for a healthy community and sustainable health system. Various approaches have been attempted in the past several decades with varying degrees of success. Health reform is a continuous process. For the United States, the largest previous success in healthcare reform was the implementation of Medicare and Medicaid. Since that time, it has been apparent that high costs and suboptimal quality outcomes require further attention. Growing interest in healthcare reform culminated in the passage of the Affordable Care Act (ACA) of 2010. The Kaiser Family Foundation has put together a summary of the Patient Protection and ACA (1). The aim of this program is to provide access to affordable health insurance, and by extension, quality healthcare to all Americans. This act required legislation regarding access to health insurance, preventive care coverage, and efficiency of healthcare. Insurance providers are required to cover certain preventive care procedures without additional costs such as copay or coinsurance to the beneficiary. On the other hand, the insured patient has shared cost responsibilities related to non-preventive services that may be recommended as part of a preventive care visit. Shared costs are viewed in part as a technique to control costs associated with overutilization of healthcare services. There are a number of informational resources available for healthcare consumers to learn about covered preventive care $(2,3)$. The ACA now provides expanded access to insurance coverage for preventive and treatment strategies to previously uninsured (4). The ACA takes on a monumental effort to address quality of care, health outcomes, and cost of care. By providing greater access to healthcare through increased numbers of insured enrollees, a cost shift is expected to take place. According to the recent health system reform legislation, insurance beneficiaries are entitled to preventive care procedures that are recommended by the U.S. Preventive Services Task Force without costsharing $(2,5,6)$. There are limitations to coverage without cost-sharing for these preventive care procedures, including but not limited to: enrollment in a participating health plan, meeting certain restriction criteria such as risk factors and gender (2,3). A listing of the current recommendations by the U.S. Preventive Services Task Force is available on the their website (7). The 45 recommended preventive services are required to be covered, as well as annual visits and the recommended prevented services covered by Medicare (5). Cost-sharing is a two-prong approach to instill personal responsibility for healthcare while controlling spending (8). However, concerns arise to the coverage and cost-sharing associated with addressing issues that arise due to preventive care. Non-preventive care services are not without costsharing, and therefore may result in elevated immediate costs to the patient. These non-preventive care costs may include ancillary tests or follow-up visits to interrogate abnormal results from preventive care visits. 


\section{Current situation}

Coverage of preventive care services aims to reduce the amount of undiagnosed or untreated conditions. This is expected to reduce costs through less invasive or complex treatment options. Cost-sharing of supplemental tests, procedures, and follow-up visits as a result of preventive care are subject to insurance deductibles or co-pays (9). The insured is then responsible for the cost of nonpreventive services. Overhauls of the healthcare system include changes to integrated delivery networks such as accountable care organizations or long-term care facilities. These changes are aimed at reducing medical costs through improved coordination and integration of care along with lowering re-admissions and healthcare acquired infections (4). These efforts are much needed, yet the impact of cost reduction remains to be determined. Furthermore, it is unclear how these cost reductions will be passed on to the patient receiving care. In addition, the assumption that the overall cost reduction from identifying underlying conditions through early intervention via preventive care visits may be flawed. The system costs may in fact be reduced but it is likely that the patient will incur early prevention costs associated with wellness efforts, medication, prophylaxis or follow-up examinations. Under the ACA, cost-sharing for basic health coverage is based on income (4). Without transparency of costs, overhead and efficiency in delivery of care, it is improbable for an average worker to afford treatment costs of a major episode. It has been reported that medical debt is a point of cause in up to $62 \%$ of bankruptcy cases (9). Of these bankruptcy cases, $75 \%$ of the debtors had health insurance (9). This points to a need for cost control and patient financial liability protection through acquired health insurance. Outof-Pocket (OOP) medical expenses can average 22,568 US dollars for a family (9). A recent report revisited this issue following Massachusetts' health reform which successfully provided insurance to previously uninsured. Unfortunately, the study did not reveal a decrease in the rate of medical bankruptcy following reform (10). Simply reducing the number of uninsured people does not result in financial solvency protection. Bankruptcy due to medical costs reflects the financial liability assumed from seeking medical intervention for diagnosis or treatment of illness. A number of factors affect the ability of the patient to pay including income resources, insurance coverage of care costs, and the cost of care. Healthcare costs remain high and annual medical expenses in excess of 20,000 US dollars are beyond the average family's financial capacity (11).

\section{Options analysis}

Many insurance companies seek to shift the financial responsibility to the consumer in order to avoid increasing health related costs (12). Even with ACA maximum limits on OOP expenses, bankruptcy remains a threat until this maximum level is brought to a manageable figure. The ACA protects consumers from catastrophic consequences of medical care. However, those in the private healthcare system face significant medical expenses when frequent visits are required to treat chronic conditions. The issue of preventive care services utilization has drawn much focus in identifying the benefits of such medical care. In general, it is accepted that services which save more money than the cost of delivering said service are of benefit. Examples include health assessments, immunizations, and preventive consultations (13). A broader definition of beneficial preventive services suggests that prevention must only result in a health benefit (13). Perhaps this is where the misinformation occurs. Prevention does not necessarily reduce medical costs as a rule, although there may be examples where cost savings occur (14). In fact, prevention costs can exceed the amount saved. There are a number of preventive services that directly reduce costs and include childhood immunizations, risky behavior counseling (e.g. smoking cessation, illicit drug abstinence), cardiovascular prophylaxis such as daily aspirin, and certain cancer screens (13). By coordinating preventive services with lifestyle changes improved public health is expected. The benefit is longer and potentially more productive lives attributed to preventive care. In order to achieve higher efficacy and therefore return on investment in preventive care, those services with minimal evidence of effectiveness should be held to an assessment process (13). This strategy will help preserve healthcare dollars for those procedures with known benefit. A downside is that emerging strategies or procedures are unable to present historical information to their potential benefit. This would become a prohibitive barrier to those treatments which would otherwise have a positive impact on healthcare.

Outside of strictly medical facilities exist additional opportunities to positively influence population health. Wellness strategies that rely on employers to provide wellness programs can encourage healthy lifestyle behaviors (15). In efforts to address concerns related to rising healthcare costs, employers are deploying strategies aimed at reducing employee absenteeism while improving production and corporate morale (15). These programs address individual behaviors including physical activity, exercise, weight loss, and smoking cessation (15). Potential hurdles companies will face include ethical, legal, and practical aspects of mandating employee participation and adherence to wellness initiatives (15). Employees may potentially feel manipulated into participating in the wellness program. Additionally, chronically ill employees may feel a burden of embarrassment or penalized by not being able to reap the benefits of a reward system. Other unintended consequences include the time constraints of busy professionals balancing work and family life and thus, are unable to participate in a corporate fitness program. Of course, the benefit to wellness programs and initiatives are the anticipated gains in employee productivity, reduced stress, happiness, emotional well-being, and overall lowered health related expenses. In order to avoid legal sanctions, employers should seek to avoid unintentional discrimination in these programs for protected groups. Appropriate alternatives should be available. Furthermore, taking action to deploy a wellness program demonstrates the willingness and character of the company to develop healthy 
and productive staff.

The goal of cost-sharing is to shift patient behavior into a consumer mind-set focused on deriving the best value for the dollar. This approach is termed value-based cost-sharing (16). In healthcare, value-based cost-sharing occurs when the patient seeks treatments, medications, procedures, and other services that yield the highest value compared with other options. In general, this has been largely applied to medications (16). Preferred providers or preventive services can result in lower payments on the part of the insurance beneficiary. Cost-sharing is one piece of a value-based formula in reshaping healthcare. In contrast, cost-sharing may reduce health due to consumer choices to avoid costly medication which has a negative impact (16). A further complication is the difficulty in determining what constitutes high-value care. The definition of value is interpreted differently among different people. Administrative costs for determining value are prohibitively high due to the complexities associated with evaluating healthcare costs (16). The need for careful policy development to avoid pitfalls of patient cost-sharing is imperative for effective performance and quality outcomes. Finally, even good values should be affordable to the patient. This leads to the need for overall reduction in costs and translating those cost reductions into affordable prices for the patient's cost share.

\section{Discussion and recommendation}

Savings related to preventive care extend beyond the immediate effect into future benefits, particularly for vaccinations (14). Preventive care options should be modeled after those procedures which have a cost which is lower than the potential treatment cost. Cost-sharing is a major component of current insurance programs, yet the incurred cost of care for a chronic or major acute condition is greater than the financial capacity of the average American family. Despite the maximum OOP limit imposed by the ACA, there remains a great deal to be done in order to offset these costs. Cost reduction for healthcare is essential. In addition, insurance providers need to assume a greater bulk of the cost of care. Healthcare reform requires continuous process improvements in access of care, quality, and cost control (17). While the ACA does much to address cost control at the institutional level, it remains unclear how these controls result in a reduction of cost at the individual patient level. More transparency of costs in addition to cost savings for those undergoing preventive treatments is necessary for successful execution of the preventive care strategy. Compliance with treatment regimens is strongly influenced by patient education as well as economical and affordable solutions. To that end, cost reduction is essential in order to reduce the patient's financial responsibility. Cost management remains a popular topic among care providers, insurers, and prospective patients. Effective cost-sharing enables a number of fully covered preventive care options to the prospective patient while avoiding barriers to essential care. The result can be reduced specialists visits without negatively impacting primary care visits (18). Cost-sharing has demonstrated effectiveness in managing healthcare costs for insurers. In order to reach the desired level of healthcare utilization and optimal outcomes, a balance between fully covered (no OOP cost) preventive care, cost-sharing, and maximum OOP limits for patients is essential. The ACA has the infrastructure in place to address these issues, but further refinement is likely necessary. Furthermore, education of insurance beneficiaries on cost-sharing exempt preventive care services will prevent these patients from avoiding care due to concerns over cost (19). There remains confusion related to the cost of services resulting in delays of care even when the cost is free or minimal. Removing barriers related to cost concerns, including education regarding coverage, is essential for early intervention of both acute and chronic illnesses. By removing even small cost barriers such as copays and OOP expenses for major preventive care services, the ACA has taken steps to identify treatment for improved health (20). It is widely evident that the U.S. has the best resources available for healthcare.

\section{Conclusions}

Rather than stymieing the adoption of solutions related to preventive costs and cost-sharing, it is incumbent that the U.S. society embrace the overarching aim to improve national health quality. There are a number of dilemmas and challenges associated with formulating a program that has the perfect solution to every combination of circumstances. Notwithstanding those challenges, it remains to be seen how the next iteration should be unfolded to address healthcare reform. It is evident that an optimal combination of covered preventive care services, cost-sharing, and cost reductions are needed to improve quality and access to care. In the interim, policy officials, healthcare payers, and healthcare professionals should synergize their efforts to provide high quality healthcare at the lowest cost to all those in need of care. Ongoing improvements will remain essential in the endeavor to deliver high quality, affordable healthcare to all Americans. Clearly, the need to increase access to these valuable resources has the potential to have a major impact on healthcare and wellness. The benefits of reaching the noble goal of healthcare access and affordability serve both present and future generations. Navigating the challenges presented to provide broad access to health resources is surmountable given the available intellectual capacity. A patient centric focus is imperative when formulating reformation solutions. Maintaining the perspective of patient first will serve as a foundation for both quality and cost concerns.

\section{Ethical issues}

Not applicable.

Competing interests

Authors declare that they have no competing interests.

Authors' contributions

RBD prepared the manuscript. AJH reviewed and provided feedback for the manuscript. 


\section{References}

1. Foundation TKF. Summary of the Patient Protection and Affordability Act [internet]. 2013 [updated 2014 June]. Available from: http://www.kff.org/healthreform/8061.cfm

2. Healthcare.gov. Preventive Care Benefits [internet]. [updated 2014 June]. Available from: https://www.healthcare.gov/whatare-my-preventive-care-benefits/\#part=1

3. HHS.gov/Healthcare. Affordable Care Act Rules on Expanding Access to Preventive ServicesforWomen [internet]. 2011 [updated 2014 June]. Available from: http://www.hhs.gov/healthcare/facts/ factsheets/2011/08/womensprevention08012011a.html

4. Molinari C. Does the accountable care act aim to promote quality, health, and control costs or has it missed the mark? Comment on "health system reform in the United States". Int $J$ Health Policy Manag 2014; 2: 97-9. doi: 10.15171/ijhpm.2014.23

5. Cassidy A. Health Policy Brief: Preventive services without cost sharing. Health Aff (Millwood) 2010. Available from: http://www.healthaffairs.org/healthpolicybriefs/brief_pdfs/ healthpolicybrief_37.pdf

6. Cogan JA. The Affordable Care Act's Preventive Services Mandate: Breaking Down the Barriers to Nationwide Access to Preventive Services. J Law Med Ethics 2011; 39: 355-65. doi: 10.1111/j.1748-720X.2011.00605.x

7. Force USPST. USPSTF $A$ and $B$ Recommendations [internet]. [cited 2014 August]. Available from: http://www. uspreventiveservicestaskforce.org/uspstf/uspsabrecs.htm

8. Saloner BP, Sabik LP, Sommers BD. Pinching the Poor? Medicaid Cost Sharing under the ACA. N Engl J Med 2014; 370: 1177-80. doi: 10.1056/NEJMp1316370

9. Himmelstein DU, Thorne D, Warren E, Woolhandler S. Medical bankruptcy in the United States, 2007: results of a national study. Am J Med 2009; 122: 741-6. doi: 10.1016/j.amjmed.2009.04.012

10. Himmelstein DU, Thorne D, Woolhandler S. Medical bankruptcy in Massachusetts: has health reform made a difference? $\mathrm{Am} J$ Med 2011; 124: 224-8. doi: 10.1016/j.amjmed.2010.11.009

11. Bucks BK, Kennickell AB, Mach TL, More KB. Changes in U.S. family finances from 2004-2007: evidence from the Survey of Consumer Finances. Federal Reserve Bulletin 2009; 92: A1-56.

12. Sugden R. Sick and (still) broke: Why the Affordable Care Act won't end medical bankruptcy. Washington University Journal of Law and Policy 2012; 38: 34.

13. Maciosek MV, Coffield AB, Flottemesch TJ, Edwards NM, Solberg LI. Greater Use Of Preventive Services In U.S. Health Care Could Save Lives At Little Or No Cost. Health Aff (Millwood) 2010; 29: 1656-60. doi: 10.1377/hlthaff.2008.0701

14. Russell LB. Prevention's potential for slowing the growth of medical spending [internet]. Washington DC, 2007. Available from: http://www.ihhcpar.rutgers.edu/downloads/ russellnchc2007.pdf

15. Cavico FJ, Mujtaba BG. Health and Wellness Policy Ethics. Int J Health Policy Manag 2013; 1: 111-3. doi: 10.15171/ ijhpm.2013.19

16. Thomson S, Schang L, Chernew ME. Value-Based Cost Sharing In The United States And Elsewhere Can Increase Patients' Use Of High-Value Goods And Services. Health Aff (Millwood) 2013; 32: 704-12. doi: 10.1377/hlthaff.2012.0964

17. McDonough JE. Health system reform in the United States. Int $J$ Health Policy Manag 2014; 2: 5-8. doi: 10.15171/ijhpm.2014.02

18. Shah ND, Naessens JM, Wood DL, Stroebel RJ, Litchy W, Wagie A, et al. Mayo Clinic Employees Responded To New Requirements For Cost Sharing By Reducing Possibly Unneeded Health Services Use. Health Aff (Millwood) 2011; 30: 2134-41. doi: $10.1377 /$ hlthaff. 2010.0348

19. Reed ME, Graetz I, Fung V, Newhouse JP, Hsu J. In ConsumerDirected Health Plans, A Majority Of Patients Were Unaware Of Free Or Low-Cost Preventive Care. Health Aff (Millwood) 2012; 31: 2641-8. doi: 10.1377/hlthaff.2012.0059

20. Johnson PA, Fitzgerald T. The Affordable Care Act and the opportunity to improve prevention in women: how to make the most of the law. Clin Chem 2014; 60: 138-40. doi: 10.1373/ clinchem.2013.203703 